\title{
ON CERTAIN PAIRS OF SURFACES IN ORDINARY SPACE
}

\author{
BUCHIN SU
}

1. Introduction. In a recent paper ${ }^{1}$ Jesse Douglas has proposed and solved the following problem: To determine the form of the linear element of a surface in ordinary space upon which exists a family of $\infty^{2}$ curves possessing two properties: (1) The angular excess of any triangle $A B C$ formed by curves of the family $\mathcal{F}$ is proportional to the area of the triangle:

$$
\varepsilon=A+B+C-\pi=k \mathcal{A},
$$

where $k$ denotes a constant; (2) The curves of $\mathcal{F}$ are a linear system; that is, a point transformation exists which converts them in to the straight lines of a plane. It is natural to inquire what class of surfaces we shall obtain if, instead of using property (2), we make the less specific demand that a point transformation exists which converts the curves of $\mathcal{F}$ in to the geodesics of another surface. Here we have found certain pairs of surfaces $S$ and $S_{1}$ which furnish the complete solution of our generalized problem. According to whether the constant $k$ is zero or not, the linear elements of $S$ and $S_{1}$ take different types, whose derivation constitutes the purpose of the present paper.

2. Conditions for the property $\varepsilon=k \mathcal{A}$. As was shown by Douglas, ${ }^{2}$ the necessary and sufficient conditions that every curve of a family $\mathcal{F}$ upon a surface $S$ should have the property $\mathcal{E}=k \mathcal{A} A$ can be expressed by the relation

$$
d s / \rho=P d u+Q d v,
$$

where $1 / \rho$ is the geodesic curvature of the curve and $P, Q$ obey the condition.

$$
Q_{u}-P_{v}=(k-K) W .
$$

For the subsequent discussion it is convenient to consider both surfaces $S$ and $S_{1}$, wherein the curves of $\mathcal{F}$ upon $S$ correspond to the geodesics of $S_{1}$. Let $(u, v)$ be general coordinates of the corresponding points on these surfaces, so that the first fundamental form of $S$ is

Received by the editors February 1, 1943.

$1 \mathrm{~J}$. Douglas, $A$ new special form of the linear element of a surface, Trans. Amer. Math. Soc. vol. 48 (1940) pp. 101-116.

${ }^{2}$ Douglas, loc. cit., p. 108. 


$$
d s^{2}=E d u^{2}+2 F d u d v+G d v^{2}
$$

and that of $S_{1}$ is

$$
d s_{1}^{2}=E_{1} d u^{2}+2 F_{1} d u d v+G_{1} d v^{2} .
$$

According to the classical theorem of Tissot, ${ }^{3}$ there exists upon $S$ one and, in general, only one orthogonal system of curves which corresponds to an orthogonal system upon $S_{1}$. Suppose that these surfaces $S$ and $S_{1}$ are referred to the orthogonal curves which correspond to each other; then we have

$$
\begin{aligned}
& d s^{2}=E d u^{2}+G d v^{2}, \\
& d s_{1}^{2}=E_{1} d u^{2}+G_{1} d v^{2} .
\end{aligned}
$$

In orthogonal coordinates $(u, v)$, the geodesic curvature $1 / \rho$ of any curve $v=v(u)$ upon the surface $S$ is given by ${ }^{4}$

$$
\begin{aligned}
& d s / \rho=(E G)^{-1 / 2}\left(E+G v^{\prime 2}\right)^{-1}\left\{E G v^{\prime \prime}-(1 / 2) E E_{v}\right. \\
& \left.+\left(E G_{u}-(1 / 2) G E_{u}\right) v^{\prime}+\left((1 / 2) E G_{v}-G E_{v}\right) v^{\prime 2}+(1 / 2) G G_{u} v^{\prime 3}\right\} d u .
\end{aligned}
$$

Therefore, for a family $\mathcal{F}$, we have by (2):

$$
v^{\prime \prime}=A+B v^{\prime}+C v^{\prime 2}+D v^{\prime 3},
$$

where

$$
\left\{\begin{array}{l}
A=(1 / 2) E_{v} / G+(E / G)^{1 / 2} P \\
B=(1 / 2) E_{u} / E-G_{u} / G+(E / G)^{1 / 2} Q \\
C=E_{v} / E-(1 / 2) G_{v} / G+(G / E)^{1 / 2} P \\
D=-(1 / 2) G_{u} / E+(G / E)^{1 / 2} Q
\end{array}\right.
$$

and

$$
Q_{u}-P_{v}=(k-K)(E G)^{1 / 2} .
$$

That is: the form (9) with additional condition (11) is characteristic of curves having the property $\varepsilon=k \mathcal{A} A$ upon the surface $S$.

3. Geodesic representation of the family $\mathcal{F}$. We now have to impose the further property on the family $\mathcal{F}$.

Since the parametric curves on the surface $S_{1}$ form an orthogonal system, the differential equation of geodesics of $S_{1}$ is found to be

${ }^{3}$ Cf. G. Darboux, Le çons sur la thêorie générale des surfaces, vol. 3, 1894, p. 47.

${ }^{4} \mathrm{Cf}$. W. Blaschke, Vorlesungen über Differentialgeometrie, 3d edition, 1930, p. 175. Write $F=0, u^{\prime}=1, u^{\prime \prime}=0$.

6 Darboux, loc. cit., p. 49. 


$$
\begin{aligned}
v^{\prime \prime}= & \frac{1}{2} \\
& \frac{\partial E_{1}}{\partial v} / G_{1}+\left(\frac{1}{2} \frac{\partial E_{1}}{\partial u} / E_{1}-\frac{\partial G_{1}}{\partial u} / G_{1}\right) v^{\prime} \\
& +\left(\frac{\partial E_{1}}{\partial v} / E_{1}-\frac{1}{2} \frac{\partial G_{1}}{\partial v} / G_{1}\right) v^{\prime 2}-\frac{1}{2}\left(\frac{\partial G_{1}}{\partial u} / E_{1}\right) v^{\prime 3}
\end{aligned}
$$

In order that they should correspond to the curves of the family $\mathcal{F}$ upon $S$, it is necessary and sufficient that the differential equations (9) and (12) be coincident with each other. This gives

$$
\left\{\begin{array}{l}
\frac{1}{2} \frac{\partial E}{\partial v} / G+(E / G)^{1 / 2} P=\frac{1}{2} \frac{\partial E_{1}}{\partial v} / G_{1}, \\
\frac{1}{2} \frac{\partial E}{\partial u} / E-\frac{\partial G}{\partial u} / G+(E / G)^{1 / 2} Q=\frac{1}{2} \frac{\partial E_{1}}{\partial u} / E_{1}-\frac{\partial G_{1}}{\partial u} / G_{1}, \\
\frac{\partial E}{\partial v} / E-\frac{1}{2} \frac{\partial G}{\partial v} / G+(G / E)^{1 / 2} P=\frac{\partial E_{1}}{\partial v} / E_{1}-\frac{1}{2} \frac{\partial G_{1}}{\partial v} / G_{1}, \\
\frac{1}{2} \frac{\partial G}{\partial u} / E-(G / E)^{1 / 2} Q=\frac{1}{2} \frac{\partial G_{1}}{\partial u} / E_{1} .
\end{array}\right.
$$

From the first and the last of these equations we have the expressions for $P$ and $Q$ :

$$
\left\{\begin{array}{l}
P=\frac{1}{2}(G / E)^{1 / 2}\left\{\frac{\partial E_{1}}{\partial v} / G_{1}-\frac{\partial E}{\partial v} / G\right\} \\
Q=\frac{1}{2}(E / G)^{1 / 2}\left\{\frac{\partial G}{\partial u} / E-\frac{\partial G_{1}}{\partial u} / E_{1}\right\}
\end{array}\right.
$$

Substitution of these expressions in the remaining equations of (13) shows that the fundamental quantities $E, G ; E_{1}, G_{1}$ are related by

$$
\left\{\begin{array}{l}
\frac{\partial}{\partial v} \log \frac{E G_{1}}{G E_{1}}=\left(1-\frac{G E_{1}}{E G_{1}}\right) \frac{\partial \log E_{1}}{\partial v} \\
\frac{\partial}{\partial u} \log \frac{E G_{1}}{G E_{1}}=\left(\frac{E G_{1}}{G E_{1}}-1\right) \frac{\partial \log G_{1}}{\partial u} .
\end{array}\right.
$$

In interpreting these conditions, it is important to distinguish the case $(E / G):\left(E_{1} / G_{1}\right)=1$ from $(E / G):\left(E_{1} / G_{1}\right) \neq 1$. If $(E / G):\left(E_{1} / G_{1}\right)=1$, then we can put

$$
E_{1}=\rho E, \quad G_{1}=\rho G,
$$


where $\rho \neq 0$, so that the surfaces $S$ and $S_{1}$ are conformal. In this case the equations (15) are satisfied identically, while the equations (14) give

$$
\left\{\begin{array}{l}
P=\frac{1}{2}(E / G)^{1 / 2} \frac{\partial \log \rho}{\partial v}, \\
Q=-\frac{1}{2}(G / E)^{1 / 2} \frac{\partial \log \rho}{\partial u} .
\end{array}\right.
$$

Substituting these expressions in (11) we have

$$
\begin{aligned}
& \frac{\partial}{\partial v}\left\{(E / G)^{1 / 2} \frac{\partial \log \rho}{\partial v}\right\}+\frac{\partial}{\partial u}\left\{(G / E)^{1 / 2} \frac{\partial \log \rho}{\partial u}\right\} \\
&+2(k-K)(E G)^{1 / 2}=0 .
\end{aligned}
$$

This condition can be interpreted geometrically as follows: by means of the formula of G. Frobenius for Gaussian curvature $^{6}$ we obtain for the surface $S$ with linear element (6)

$$
K=-\frac{1}{2 W}\left\{\frac{\partial}{\partial v}\left(E_{v} / W\right)+\frac{\partial}{\partial u}\left(G_{u} / W\right)\right\},
$$

where $W=(E G)^{1 / 2}$, and similarly, for the surface $S_{1}$ with linear element (7)

$$
K_{1}=-\frac{1}{2 W_{1}}\left\{\frac{\partial}{\partial v}\left(\frac{\partial E_{1}}{\partial v} / W_{1}\right)+\frac{\partial}{\partial u}\left(\frac{\partial G_{1}}{\partial u} / W_{1}\right)\right\},
$$

where $W_{1}=\left(E_{1} G_{1}\right)^{1 / 2}$.

If the expressions of $E_{1}$ and $G_{1}$ given by (16) are substituted in the right-hand member of (20), then

$$
\begin{aligned}
K_{1}= & -\frac{1}{2 \rho W}\left\{\frac{\partial}{\partial v}\left(E_{v} / W\right)+\frac{\partial}{\partial u}\left(G_{u} / W\right)\right\} \\
& -\frac{1}{2 \rho W}\left\{\frac{\partial}{\partial v}\left((E / G)^{1 / 2} \frac{\partial \log \rho}{\partial v}\right)+\frac{\partial}{\partial u}\left((G / E)^{1 / 2} \frac{\partial \log \rho}{\partial u}\right)\right\} .
\end{aligned}
$$

A reference to (18) and (19) gives immediately the relation

$$
\rho K_{1}=k,
$$

which is, of course, equivalent to (18).

If $k \neq 0$, then we have

\footnotetext{
${ }^{6}$ Blaschke, loc. cit., p. 117.
} 


$$
\left\{\begin{array}{l}
d s^{2}=\left(K_{1} / k\right)\left(E_{1} d u^{2}+G_{1} d v^{2}\right), \\
d s_{1}^{2}=E_{1} d u^{2}+G_{1} d v^{2} .
\end{array}\right.
$$

Therefore the surface $S_{1}$ may be arbitrarily selected, the only restriction being that it is non-developable, and any surface $S$, necessarily conformal to $S_{1}$, with the linear element

$$
d s^{2}=\left(K_{1} / k\right) d s_{1}^{2}
$$

possesses the two stated properties, where $K_{1}$ is the Gaussian curvature of $S_{1}$ and $k$ a constant different from zero. In particular when a surface of constant Gaussian curvature $k$ is taken for $S_{1}$, the corresponding surface $S$ is applicable to $S_{1}$.

This result not only proves the existence but also furnishes a remarkable class of the surfaces under consideration.

On the contrary, if $k=0$ in the relation (21), then $K_{1}$ must necessarily vanish, because $\rho$ is by no means zero, so that the surface $S_{1}$ is developable. In this case, the surface $S$ is arbitrary and $\mathcal{F}$ must be a conformal image of the $\infty^{2}$ straight lines of a plane. That no other family of curves upon a generic surface $S$ can be linear and such that the sum of the angles in every triangle of curves in the family is two right angles has been proved analytically by E. Kasner ${ }^{7}$ and synthetically by J. Douglas. ${ }^{8}$

We now consider the case where

$$
(E / G):\left(E_{1} / G_{1}\right) \neq 1 \text {. }
$$

The partial differential equations (15) are easily integrated, and the result may be written in the form

$$
\left(E G_{1}\right) /\left(G E_{1}\right)=1-E_{1} U^{2}, \quad\left(G E_{1}\right) /\left(E G_{1}\right)=1+G_{1} V^{2},
$$

where $U$ denotes any function of $u$ alone, and $V$ any function of $v$ alone. The assumption (24) shows that neither $U$ nor $V$ is zero.

From (25) follows the relation

$$
E_{1} U^{2}\left(G_{1} V^{2}+2\right)=G_{1} V^{2}\left(2-E_{1} U^{2}\right) .
$$

It may happen that both members of (26) are zero. Since $E_{1}, G_{1}, U, V$ are different from zero, we have in this case

' E. Kasner, A characteristic property of isothermal systems of curves, Math. Ann. vol. 59 (1904) pp. 352-354.

${ }^{8} \mathrm{~J}$. Douglas, $A$ criterion for the conformal equivalence of a Riemann space to a Euclidean space, Trans. Amer. Math. Soc. vol. 27 (1925) pp. 299-306. 


$$
E_{1}=2 U^{-2}, \quad G_{1}=-2 V^{-2},
$$

so that

$$
E / G=V^{2} / U^{2}
$$

The expressions (14) now become

$$
P=-\frac{1}{2} \frac{V}{U} \frac{\partial \log E}{\partial v}, \quad Q=\frac{1}{2} \frac{U}{V} \frac{\partial \log G}{\partial u} .
$$

Substituting them in (18) and remembering the formula (19) in addition to the relation (28), we obtain

$$
k=0 .
$$

That is, the sum of the angles in every triangle formed by three curves of the family $\mathcal{F}$ under consideration must be two right angles, and the linear elements of the surfaces $S$ and $S_{1}$ are, after a suitable transformation of the type

$$
\bar{u}=\phi(u), \quad \bar{v}=\psi(v),
$$

reducible to the form

$$
\left\{\begin{array}{l}
d s^{2}=\lambda(u, v)\left(d u^{2}+d v^{2}\right), \\
d s_{1}^{2}=d u^{2}-d v^{2}
\end{array}\right.
$$

It remains for us to consider the case

$$
\left(2-E_{1} U^{2}\right)\left(G_{1} V^{2}+2\right) \neq 0 .
$$

Setting (26) in the form

$$
\left(E_{1} U^{2}\right) /\left(2-E_{1} U^{2}\right)=\left(G_{1} V^{2}\right)\left(G_{1} V^{2}+2\right)=\tau,
$$

we obtain

$$
E_{1}=2 \tau(1+\tau)^{-1} U^{-2}, \quad G_{1}=2 \tau(1-\tau)^{-1} V^{-2}
$$

and

$$
(E / G)^{1 / 2}=(1-\tau)(1+\tau)^{-1} V U^{-1} .
$$

The expressions (14) for $P$ and $Q$ may be written in the form

$$
\left\{\begin{array}{l}
P=\frac{1}{2}\left\{\frac{V}{U} \frac{\partial \log E_{1}}{\partial v}-\frac{1}{W} \frac{\partial E}{\partial v}\right\}, \\
Q=-\frac{1}{2}\left\{\frac{U}{V} \frac{\partial \log G_{1}}{\partial u}-\frac{1}{W} \frac{\partial G}{\partial u}\right\} .
\end{array}\right.
$$


Consequently, we have

$$
\begin{gathered}
Q_{u}-P_{v}=-\frac{1}{2 V} \frac{\partial}{\partial u}\left(U \frac{\partial}{\partial u} \log G_{1}\right)-\frac{1}{2 U} \frac{\partial}{\partial v}\left(V \frac{\partial}{\partial v} \log E_{1}\right) \\
+\frac{1}{2}\left\{\frac{\partial}{\partial u}\left(G_{u} / W\right)+\frac{\partial}{\partial v}\left(E_{v} / W\right)\right\},
\end{gathered}
$$

namely,

$$
\begin{aligned}
Q_{u}-P_{v}= & -\frac{1}{2 V} \frac{\partial}{\partial u}\left(U \frac{\partial}{\partial u} \log G_{1}\right) \\
& -\frac{1}{2 U} \frac{\partial}{\partial v}\left(V \frac{\partial}{\partial v} \log E_{1}\right)-W K,
\end{aligned}
$$

as may easily be seen on account of (19). By comparison of (38) with (11) it follows that

$$
2 k(E G)^{1 / 2}=-\frac{1}{V} \frac{\partial}{\partial u}\left(U \frac{\partial}{\partial u} \log G_{1}\right)-\frac{1}{U} \frac{\partial}{\partial v}\left(V \frac{\partial}{\partial v} \log E_{1}\right) .
$$

In virtue of (35) we reach the relation:

$$
2 k(E G)^{1 / 2}=-\frac{1}{U V}\left[\left(U \frac{\partial}{\partial u}\right)^{2} \log \frac{\tau}{1-\tau}+\left(V \frac{\partial}{\partial v}\right)^{2} \log \frac{\tau}{1+\tau}\right] .
$$

It is important to distinguish the case $k=0$ from $k \neq 0$. The latter case is of more interest; we find by means of (40) an additional relation between $E$ and $G$, namely,

$$
(E G)^{1 / 2}=-(1 / 2 k U V)\left[\left(U \frac{\partial}{\partial u}\right)^{2} \log \frac{\tau}{1-\tau}+\left(V \frac{\partial}{\partial v}\right)^{2} \log \frac{\tau}{1+\tau}\right] .
$$

Therefore the linear elements of $S$ and $S_{1}$ are, from (35), (36) and (41), given by

$$
\left\{\begin{aligned}
d s^{2}= & -\frac{1}{2 k}\left[\left(U \frac{\partial}{\partial u}\right)^{2} \log \frac{\tau}{1-\tau}+\left(V \frac{\partial}{\partial v}\right)^{2} \log \frac{\tau}{1+\tau}\right] \\
& \cdot\left[\frac{1-\tau}{1+\tau} U^{-2} d u^{2}+\frac{1+\tau}{1-\tau} V^{-2} d v^{2}\right], \\
d s_{1}^{2}= & \frac{2 \tau}{1+\tau} U^{-2} d u^{2}+\frac{2 \tau}{1-\tau} V^{-2} d v^{2},
\end{aligned}\right.
$$

where $\tau$ denotes an arbitrary function of $u, v$.

If the parameters $u, v$ are subjected to a suitable transformation 
of type (31), then the pair of surfaces $S$ and $S_{1}$ is characterized by the linear elements of a special form:

$$
\left\{\begin{array}{l}
d s^{2}=-\frac{1}{2 k}\left\{\frac{\partial^{2}}{\partial u^{2}} \log \frac{\tau}{1-\tau}+\frac{\partial^{2}}{\partial v^{2}} \log \frac{\tau}{1+\tau}\right\}\left\{\frac{1-\tau}{1+\tau} d u^{2}+\frac{1+\tau}{1-\tau} d v^{2}\right\} \\
d s_{1}^{2}=\frac{2 \tau}{1+\tau} d u^{2}+\frac{2 \tau}{1-\tau} d v^{2}
\end{array}\right.
$$

involving an arbitrary function $\tau$ of $u$ and $v$.

If $k=0$, then (39) becomes, by applying a transformation of type (31),

$$
\frac{\partial^{2}}{\partial v^{2}} \log E_{1}+\frac{\partial^{2}}{\partial u^{2}} \log G_{1}=0
$$

Therefore we obtain

$$
\left\{\begin{array}{l}
d s_{1}^{2}=\left(E^{-1 / 2}-G^{-1 / 2}\right)\left(E^{1 / 2} d u^{2}+G^{1 / 2} d v^{2}\right) \\
d s^{2}=E d u^{2}+G d v^{2}
\end{array}\right.
$$

where the quantities $E$ and $G$ are related by

$$
\frac{\partial^{2}}{\partial v^{2}} \log \left(1-(E / G)^{1 / 2}\right)+\frac{\partial^{2}}{\partial u^{2}} \log \left((G / E)^{1 / 2}-1\right)=0 .
$$

Thus the problem of determining the form of linear elements of a pair of surfaces $S$ and $S_{1}$ with the stated properties is completely solved.

It should be observed that the analogous problem for higher dimensional spaces may be of some interest. We hope to consider it in a future paper.

Meitan, Kweichow, China 